\title{
Sliding Mode with Adaptive Control of Robot Manipulator Trajectory Tracking using Neural Network Approximation
}

\author{
Monisha Pathak, Mrinal Buragohain
}

\begin{abstract}
This paper briefly discusses about the Robust Controller based on Adaptive Sliding Mode Technique with RBF Neural Network (ASMCNN) for Robotic Manipulator tracking control in presence of uncertainities and disturbances. The aim is to design an effective trajectory tracking controller without any modelling information. The ASMCNN is designed to have robust trajectory tracking of Robot Manipulator, which combines Neural Network Estimation with Adaptive Sliding Mode Control. The RBF model is utilised to construct a Lyapunov function-based adaptive control approach. Simulation of the tracking control of a 2dof Robotic Manipulator in the presence of unpredictability and external disruption demonstrates the usefulness of the planned ASMCNN.
\end{abstract}

Keywords: Sliding Mode Control, Robot manipulator, Trajectory Tracking, Neural Network.

\section{INTRODUCTION}

$\mathrm{O}_{\mathrm{n}}$ ne of the most prominent intelligent computation systems is the neural network, which has an innate learning capability and can estimate any nonlinear continuing function with precision. Many adaptive neural network control strategy have been created to design robust tracking control of robot manipulators with high nonlinearity using the universal estimation property of many layer neural networks. Sliding mode control (SMC) is one of the most powerful robust non-linear control technique for robot manipulators tracking control in the presence of parametric unpredictability and external disruption [5,6,9]. Sliding mode control necessitates information on the top bound of model unpredictability and external disruptions, and there is always chattering present in practical applications.

Robotic manipulator is a nonlinear, uncertain complex system with unknown dynamics. Designing a robust controller for robot manipulator trajectory tracking is a very challenging task.[6]. Uncertainties are always present due to nonlinear frictions and joints flexibility. Many research approaches e.g. Neural network (NN) [1,3,4,15],

Manuscript received on July 24, 2021.

Revised Manuscript received on August 19, 2021.

Manuscript published on August 30, 2021.

* Correspondence Author

Monisha Pathak*, Department of Instrumentation Engineering, Jorhat Engineering College, Jorhat, Assam, India. Email:mamup@yahoo.co.in

Dr. Mrinal Buragohain, Department of Electrical Engineering, Jorhat Engineering College, Jorhat, Assam, India. Email:mrinalburagohain@gmail.com

(c) The Authors. Published by Blue Eyes Intelligence Engineering and Sciences Publication (BEIESP). This is an open access article under the CC BY-NC-ND license (http://creativecommons.org/licenses/by-nc-nd/4.0/)

Sliding-mode control [5,9,10], Adaptive control [7], and fuzzy logic $[12,14]$ are available to handle such uncertainties.

In this work a sliding mode with adaptive control based on neural network is designed for trajectory tracking of robot manipulator with modelling uncertainties. The goal is to construct a robust stable controller without using any modelling data. The RBF neural network approximates the unknown nonlinearities in the system and the weight values are adjusted online based on adaptive laws to control the trajectory. The Lyapunov function is utilised to develop the adaptive control law based on the RBF model. The sliding mode function can be thought of as a real-valued effective small. The chattering is satisfactorily minimized and the acking error approaches zero asymptotically. ASMCNN controller is designed. Also the stability study of the regulated system is performed in this Section. To validate the control scheme, simulation results conducted on a 2 DOF robotic manipulator are shown in Section IV and conclusions are given in Section $\mathrm{V}$.

\section{PROBLEM DESCRIPTION}

Let the n-link robot manipulator dynamical equation. ([16]) as

$$
A(q) \ddot{q}+C(q, \dot{q}) \dot{q}+G(q)+F(\dot{q})+\tau_{d}=\tau
$$

where $q, \dot{q}$ and $\ddot{q} \in R^{n}$ are vectors of angular position, angular velocity and angular acceleration of the joints respectively. In addition, $A(q) \in R^{n \times n}$ stands for the inertia matrix, and $C(q, \dot{q}) \dot{q} \in R^{n}$ stands for the centrifugal Coriolis torque vector. Furthermore, $G(q) \in R^{n} \quad$ stands for the gravitational vector, $F(\dot{q})$ for friction, $\tau \in R^{n}$ for joint torque vector, and

$\tau_{d}$ for unknown disturbance.

The dynamical equation of robot manipulator given in (1) has the following properties.

Property I :

The inertia matrix $A(q)$ is positive definite and symmetric for all $q \in R^{n}$ i.e, $A(q)=A(q)^{T}$ and $A(q)>0$ and it is lower and upper bounded i.e.,

$$
\varrho_{1} I \leq A(q) \leq \varrho_{2} I
$$

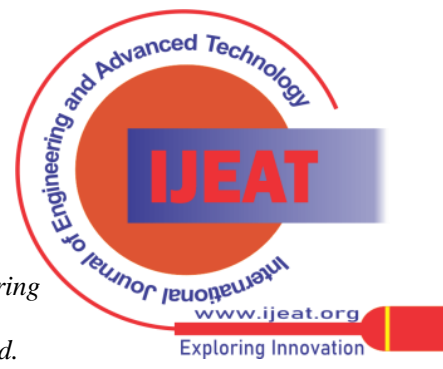




$$
m_{1} \leq\|A(q)\| \leq m_{2}
$$

where $\varrho_{1}$ and $\varrho_{2}$ are scalar values that may be calculated for any given robot arm. Likewise, the inverse of the inertia matrix is bounded since

$$
\frac{1}{\varrho_{2}} I \leq A(q)^{-1} \leq \frac{1}{\varrho_{1}} I
$$

Property II :

Matrix $\dot{A}(q)-2 C(q, \dot{q})$ is a skew symmetric matrix. i.e.,

$$
x^{T}\left[\frac{1}{2} \dot{A}(q)-C(q, \dot{q})\right] x=0, \quad \forall x \neq 0
$$

Assumption 1: The robotic manipulator's joints are all revolute.Property 1 is valid as a result of this assumption.

Assumption 2: $\quad q, \dot{q}$ and $\ddot{q} \in R^{n}$ are continuous and bounded.

\section{ASMCNN DESIGN AND STABILITY ANALYSIS}

The design of ASMCNN is discussed in this section. A trajectory tracking control aim is to design a control torque $\tau$, for a given desired trajectory $q_{d}$, the tracking error

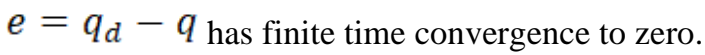

Let us define the sliding function as[8]

$$
\sigma=\dot{e}-\Lambda e
$$

where $\Lambda=\Lambda^{T}>0$, then

$\dot{q}=-\sigma+\dot{q}_{d}+\Lambda e$

$A \tilde{\sigma}=A\left(\ddot{q}_{d}-\ddot{q}+\Lambda \dot{e}\right)$

$=A\left(\ddot{q}_{d}+\Lambda \dot{e}\right)-A \ddot{q}$

$=A\left(\ddot{q}_{d}+\Lambda \dot{e}\right)+C \dot{q}+G+F+\tau_{d}-\tau$

$=A\left(\ddot{q}_{d}+\Lambda \dot{e}\right)-C \sigma+C\left(\dot{q}_{d}+\Lambda e\right)+G+F+\tau_{d}-\tau$

$=-C \sigma-\tau+\psi+\tau_{d}$

Where $\psi(\mathrm{x})=A \ddot{q}_{r}+C \dot{q}_{r}+G+F$ and $\dot{q}_{r}=\dot{q}_{d}+\Lambda e_{\text {where }}$ $\psi(\mathrm{x})$ contains all the information of modelling. RBFNN is used to approximate $\psi(\mathrm{x})$. RBFNN algorithm is given as

$$
\begin{aligned}
& h_{j}=\exp \frac{\left\|x-c_{j}\right\|^{2}}{b_{j}^{2}} \quad, j=1,2, \ldots \ldots ., m \\
& \Psi(\mathrm{x})=Z^{T} h+\varepsilon
\end{aligned}
$$

here $\mathrm{Z}$ is optimum weight value, $\mathrm{x}$ is input of RBFNN, $h=\left[h_{1} h_{2} \ldots \ldots h_{m}\right]^{T}$ and $\varepsilon$ is a very small number.

The RBFNN output is used to estimate $\psi(\mathrm{x})$.

$$
\widehat{\psi}(\mathrm{x})=\hat{Z}^{T} h
$$

where $\tilde{Z}=Z-\hat{Z}, \quad\|Z\|_{F} \leq Z_{\max }$

From (7) and (8), we have

$$
\psi-\widehat{\Psi}=Z^{T} h+\varepsilon-\hat{Z}^{T} h=\tilde{Z}^{T} h+\varepsilon
$$

Now from $\psi(\mathrm{x})$, the RBFNN input is chosen as

$$
x=\left[\begin{array}{lllll}
e^{T} & \dot{e}^{T} & q_{d}^{T} & \dot{q}_{d}^{T} & \ddot{q}_{d}^{T}
\end{array}\right]
$$

The control law for the system (1) is proposed as

$$
\tau=\widehat{\psi}(x)+K_{V} \sigma-v
$$

with $v=-\left(\varepsilon_{N}+b_{d}\right) \operatorname{sgn}(\sigma)$, where $\widehat{\Psi}(\mathrm{x})$ is estimation of $\Psi(\mathrm{x})$, and $v$ is the robust term.

The RBFNN adaptive law is formulated as

$$
\hat{\dot{Z}}=\Gamma h \sigma^{T}
$$

where $\Gamma=\Gamma^{T}>0$.

From (6) yields

$$
\begin{gathered}
A \dot{\sigma}=-C \sigma-\left(\hat{\psi}(x)+K_{V} \sigma-v\right)+\Psi+\tau_{d} \\
=-\left(K_{V}+C\right) \sigma+\tilde{Z}^{T} h+\left(\varepsilon+\tau_{d}\right)+v \\
=-\left(K_{V}+C\right) \sigma+\varsigma_{1}
\end{gathered}
$$

where $\varsigma_{1}=\tilde{Z}^{T} \varphi+\left(\varepsilon+\tau_{d}\right)+v$

The lyapunov function is used to determine stability as follows,

$$
\begin{aligned}
& V=\frac{1}{2} \sigma^{T} A \sigma+\frac{1}{2} \operatorname{t\sigma }\left(\tilde{Z}^{T} \Gamma^{-1} \tilde{Z}\right) \\
& \dot{V}=\sigma^{T} A \dot{\sigma}+\frac{1}{2} \sigma^{T} \dot{A} \sigma+t \sigma\left(\tilde{Z}^{T} \Gamma^{-1} \tilde{Z}\right) \\
& \dot{V}=-\sigma^{T} K_{V} \sigma+\frac{1}{2} \sigma^{T}(\dot{A}-2 C) \sigma+t \sigma \tilde{Z}^{T}\left(\Gamma^{-1} \tilde{\tilde{Z}}+h \sigma^{T}\right)+\sigma^{T}\left(\varepsilon+\tau_{d}+v\right) \\
& \dot{V}=-\sigma^{T} K_{V} \sigma+\sigma^{T}\left(\varepsilon+\tau_{d}+v\right) \\
& \text { Consider }
\end{aligned}
$$

$$
\begin{aligned}
& \sigma^{T}\left(\varepsilon+\tau_{d}+v\right)=\sigma^{T}\left(\varepsilon+\tau_{d}\right)+\sigma^{T}\left(-\left(\varepsilon_{N}+b_{d}\right) \operatorname{sgn}(\sigma)\right) \\
& =\sigma^{T}\left(\varepsilon+\tau_{d}\right)-\|\sigma\|\left(\varepsilon_{N}+b_{d}\right) \leq 0
\end{aligned}
$$

Finally yields

$$
\dot{V} \leq-\sigma^{T} K_{V} \sigma \leq 0
$$

\section{SIMULATION RESULTS}

Let the n-link robot manipulator dynamical equation as

$$
\begin{aligned}
& A(q) \ddot{q}+C(q, \dot{q}) \dot{q}+G(q)+F(\dot{q})+\tau_{d}=\tau \\
& A(q)=\left[\begin{array}{cc}
m_{1}+m_{2}+2 m_{3} \cos q_{2} & m_{2}+m_{3} \cos q_{2} \\
m_{2}+m_{3} \cos q_{2} & m_{2}
\end{array}\right] \\
& C(q, \dot{q})=\left[\begin{array}{cc}
-m_{3} \dot{q}_{2} \sin q_{2} & -m_{3}\left(\dot{q}_{1}+\dot{q}_{2}\right) \sin q_{2} \\
m_{3} \dot{q}_{1} \sin q_{2} & 0
\end{array}\right] \\
& G(q)=\left[\begin{array}{c}
m_{4} g \cos q_{1}+m_{5} g \cos \left(q_{1}+q_{2}\right) \\
m_{5} g \cos \left(q_{1}+q_{2}\right)
\end{array}\right]
\end{aligned}
$$

Let the disturbance $\tau_{d}=\left[\begin{array}{ll}0.2 \sin (t) & 0.2 \sin (t)\end{array}\right]^{T}$ is added to the system. The frictional force is given as $F(\dot{q})=0.02 \operatorname{sgn}(\dot{q})$. The desired trajectory [9] is

$$
q_{d 1}=1.25-1.4 e^{-t}+0.35 e^{-4 t}
$$$$
q_{d 2}=1.25+e^{-t}-0.25 e^{-4 t}
$$
$\begin{array}{llr}\text { The } & \text { initial state } & \text { is } \\ 09,0,-0.09,0] & & \text { and }\end{array}$ $m=\left[m_{1}, m_{2}, m_{3}, m_{4}, m_{5}\right]=[2.9,0.76,0.87,3.04,0.87]$

$z=\left[\begin{array}{ll}e & \dot{e}\end{array}\right]$ is the input for the RBFNN. The weight value is set to zero at the start. Gaussian function parameters are:

$$
b_{i}=10, c_{i}=\left[\begin{array}{lllllll}
-1.5 & -1 & -0.5 & 0 & 0.5 & 1 & 1.5
\end{array}\right]
$$

The following are the control law (9) and adaptive law (10) parameters:

$K_{V}=\operatorname{diag}\{20,20\}, \Gamma=\operatorname{diag}\{17,17\}, \Lambda=\operatorname{diag}\{5,5\}$. The figures $1,2,3$ and 4 shows the simulation results. Figures show both transient and steadystate characteristics for each joint. Convergence is found satrisfactory.

Published By:

Blue Eyes Intelligence Engineering and Sciences Publication

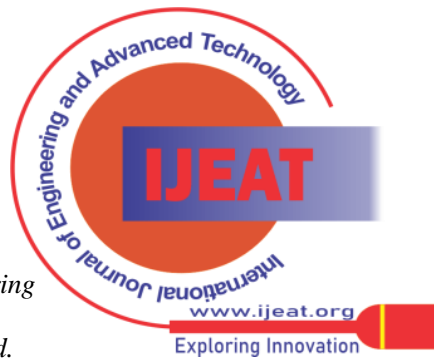




\section{CONCLUSION}

The Robust Controller based on Adaptive Sliding Mode Control with RBF Neural Network (ASMCNN) for Robot Manipulator trajectory tracking in the presence of unpredictabilitys and disturbances is briefly discussed in this research. The controller is designed with RBF Neural Network estimation without having any modelling information of the plant. The RBF model is utilised to design a Lyapunov function-based adaptive control approach. The chattering action of SMC has been successfully decreased. To validate the designed controller it is successfully employed for tracking control task of a 2dof robot manipulator in presence of uncertainities and disruptions. The tracking error approaches zero asymptotically.

\section{REFERENCES}

1. FengG.,A compensating scheme for robot tracking based on neural networks Robotics and Autonomous Systems 1995;15:199206.

2. KimYH,LewisFL,DawsonDM.,Intelligent optimal control of robotic manipulators using neural networks. Automatica 2000;36:135564.

3. WangLY,ChaiTY,ZhaiLF,.Neural-network-based terminal sliding-modecontrol of robotic manipulators including actuator dynamics. IEEE Transac- tions onIndustrial Electronics 2009;56:3296304.

4. Zuo Y,WangY,LiuX,YangSX,HuangL,WuX,etal,Neural network robustH1 tracking control strategy for robot manipulators. Applied Mathematical Modelling 2010;34:182338.

5. Utkin, V.I., Guldner, J., and Shi, J. (2009).Sliding Mode Control in Electro mechanical Systems.,London, UK: Taylor /and Francis Publishers, pp. 115-130

6. Frank L.Lewis,Darren M.Dawson,Chaouki T.Abdallah, Robot manipulator Control Theory and Practice ,2004 by Marcel Dekker.

7. Yuzheng Guo,Peng-Yung Woo, An Adaptive Fuzzy Sliding Mode Controller for Robotic ManipulatorsIEEE Transactions ,Systems and Humans, Vol. 33, NO. 2, 2003

8. F.L. Lewis, K. Liu, A. Yesildirek, Neural net robot controller with guaranteed tracking performance, IEEE Trans. Neural Network. 6 (3) (1995) 703-715.

9. S. Mondal and C. Mahanta, Adaptive Second order Terminal Sliding Mode Controller for Robotic Manipulators,Journal of the Franklin Institute, Elsevier.Journal of the Franklin Institute(Elsevier), vol. 351 issue 4, April 2014, pp. 2356 - 2377.

10. D Gao, Z Sun, W Wang ,Adaptive fuzzy sliding mode control for robotic manipulators, Control and Automation (WCICA),IEEE 2010

11. Kuo-Ching Chiou, Shiuh-Jer Huang, An adaptive fuzzy controller for robot manipulators, Mechatronics 15 (2005) 151177, Elsevier.

12. V Nekoukar, A Erfanian, Adaptive fuzzy terminal sliding mode controlfor a class of MIMO uncertain nonlinear systems,Fuzzy Sets and Systems- 179 (2011) 3449,Elsevier.

13. S Yu, X Yu, B Shirinzadeh, Z Man, Continuous finite-time control for robotic manipulators with terminal sliding mode, Automatica,41 (11), 1957-1964, 2005, Elsevier.

14. Chuan-Kai Lin,Nonsingular Terminal Sliding Mode Control of Robot Manipulators Using Fuzzy Wavelet NetworksIEEE Transactions on Fuzzy Systems (Volume:14, Issue: 6 ),849 - 859,2006

15. Wang Y, Sun W, Xiang Y, Miao S Neural network-based robus tracking control for robotsInt J Intel Autom Soft Comput 15(2), 211 222,2009

16. Spong,M.W., and Vidyasagar,M., 1989,Robot Dynamics and Control(New York: Wiley).

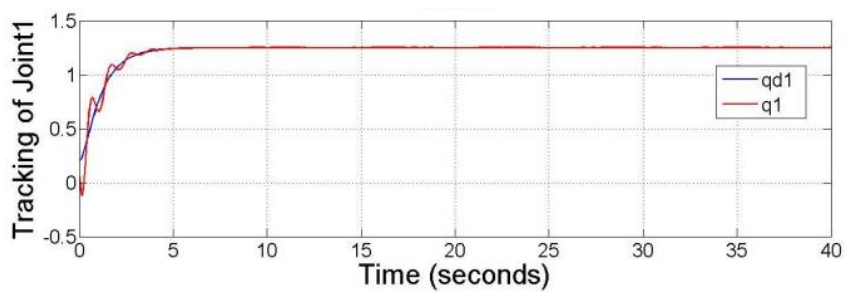

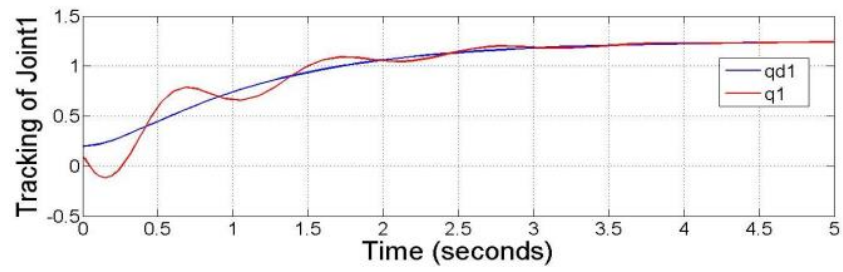

Fig. 1. Trajectory tracking of Joint1
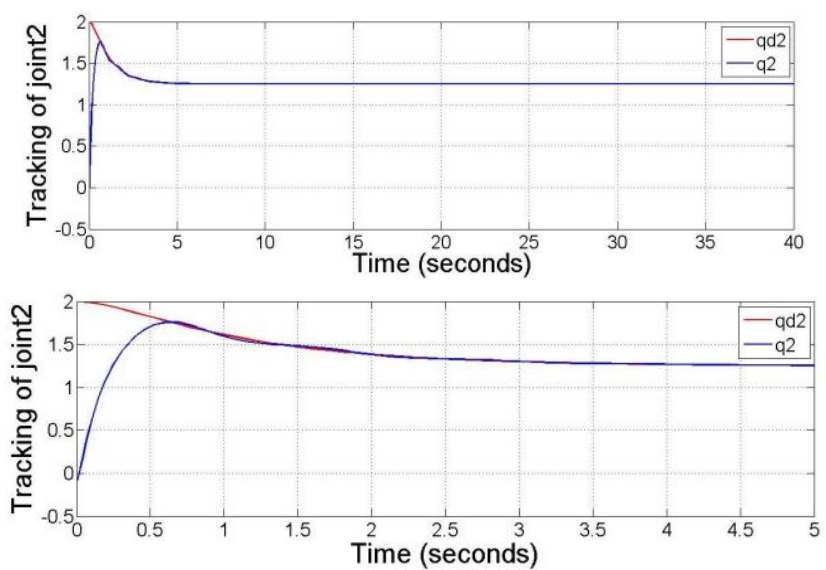

Fig. 2. Trajectory tracking of Joint2
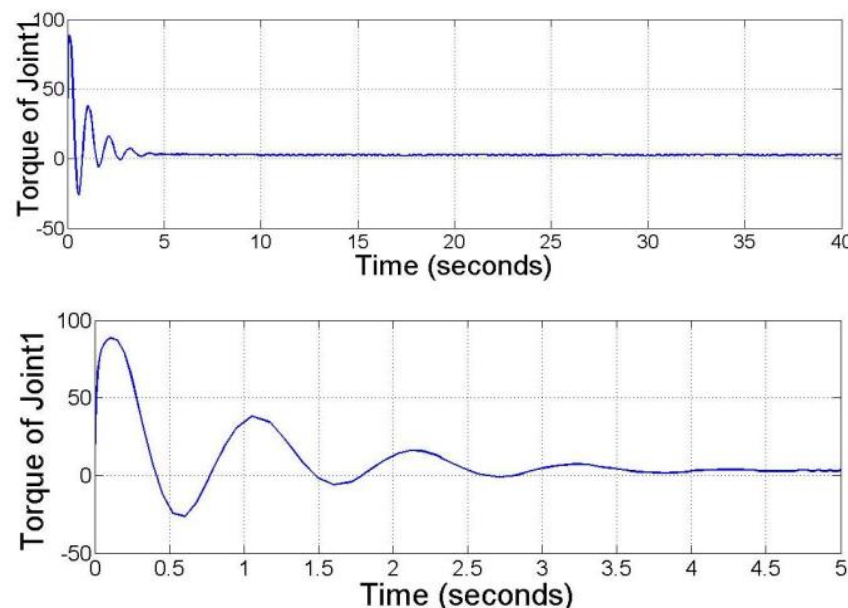

Fig. 3. Torque of Joint1
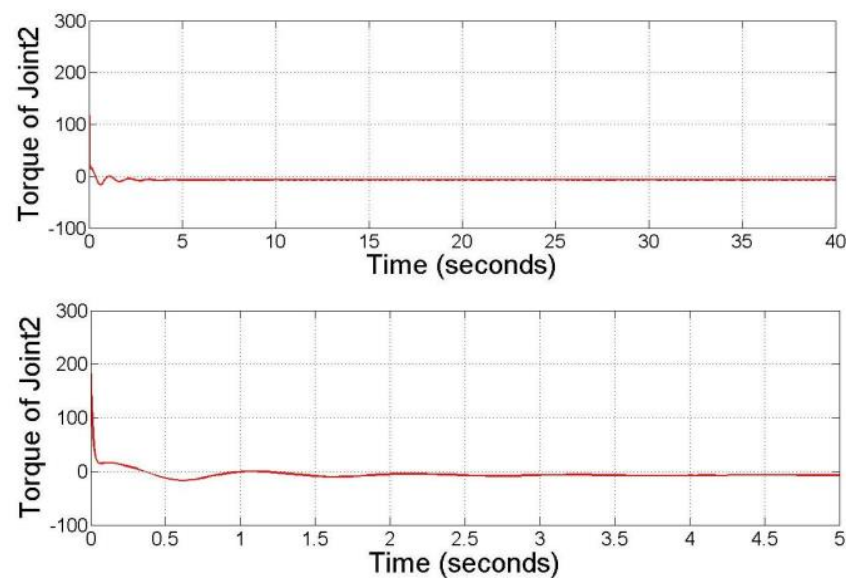

Fig. 4. Torque of Joint2

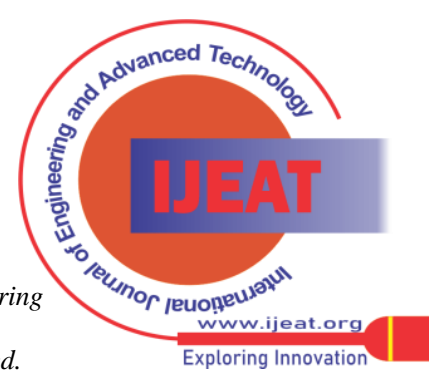




\section{AUTHORS PROFILE}

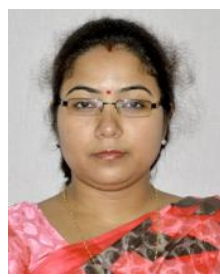

Monisha Pathak, Assistant Professor, Instrumentation Engineering Department, Jorhat Engineering College, Jorhat, Assam, India, mamup@yahoo.co.in BE: Jorhat Engineering College, Dibrugarh University. MTech: Power and Control..IIT Guwahati.. Research Interests: Instrumentation and Control, Nonlinear Control, Intelligent control.

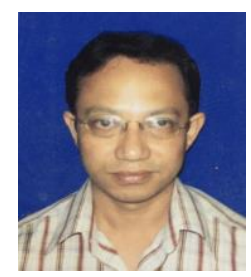

Dr. Mrinal Buragohain, Professor, Department of Electrical Engineering, Jorhat Engineering College, Jorhat received B.E degree in EE from Assam Engineering College affiliated to Gauhat University, Guwahati, Assam. M.E Degree in High Voltage Engineering from Jadavpur University, Kolkatta. After this he completed his Ph.D. in Control System from the ECE Department in the Indian Institute of Technology, Guwahati. His research interests include soft computing application in nonlinear systems and Optimal and Adaptive Control.

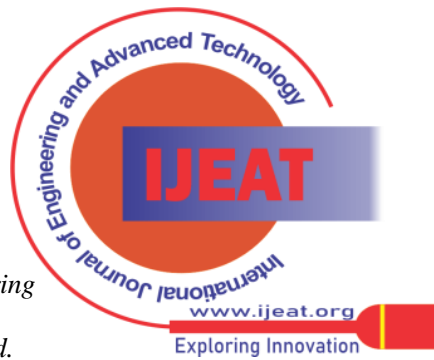

